\title{
Significance of radial artery anomalies in coronary artery bypass graft surgery
}

\author{
Chee Fui Chong, FRCSEd(CTh), MD (Lond), a and Anthony De Souza, FRCS(CTh), Brunei, Darussalam; London, England
}

$\mathrm{R}$ adial artery (RA) grafts have been reported to have significantly better early graft patency and endothelial function than long saphenous vein grafts. ${ }^{1}$ With increasing RA harvesting, knowledge of anatomic anomaly of RA can be useful for surgeons involved in harvesting RAs. We present a case of a rare RA anatomic anomaly in the forearm of a patient undergoing coronary artery bypass graft surgery.

\section{Clinical Summary}

A 45-year-old man with severe triple-vessel coronary artery disease affecting the left anterior descending (proximal stenosis $>90 \%$ ), circumflex (proximal $>75 \%$ ), and small nondominant right coronary arteries was referred for coronary artery bypass graft surgery. The preoperative bilateral modified Allen's test result was negative.

During surgery, the patient's left RA was harvested simultaneously with the internal thoracic artery and a segment of the long saphenous vein. A longitudinal curvilinear skin incision was made on the left forearm and the brachioradialis muscle reflected to expose the RA. The RA was harvested with the 2 adjacent venae comitantes by sharp dissection beginning from the elbow end. All side branches were clipped and divided. Two thirds of the way down the forearm, a large branch exiting posteriorly from the pedicle and diving deep to the pronator teres muscle was noted (Figure 1). This large branch was clipped and divided.

When the RA was completely dissected and divided at both ends, it was not possible to cannulate the proximal end of the RA. Division of the fascia covering the proximal end revealed a strand of tissue between the venae comitantes, which was continuous with the distal third of RA. On closer examination, the RA lumen was found to be continuous with that of the larger posterior branch, indicating that the RA has taken an abnormal anatomic course deep to the pronator teres muscle (Figure 1). The RA pedicle harvested was too short $(\sim 7$ $\mathrm{cm}$ ) and was grafted onto the first diagonal 1 instead of the intended obtuse marginal 1 under cardiopulmonary bypass and intermittent cold blood cardioplegic arrest. The long saphenous vein grafts were anastomosed to the posterolateral circumflex and obtuse mar-

From the Raja Isteri Pengiran Anak Saleha Hospital, ${ }^{\mathrm{a}}$ Brunei, Darussalam; and Department of Cardiac Surgery, Royal Brompton and Harefield NHS Trust, ${ }^{\mathrm{b}}$ London, England.

Received for publication Sept 29, 2007; accepted for publication Nov 15, 2007.

Address for reprints: Chee Fui Chong, PO Box 529, Bandar Seri Begawan BS8671, Brunei Darussalam (E-mail: chong_chee_fui@hotmail.com).

J Thorac Cardiovasc Surg 2008;135:1389-90

$0022-5223 / \$ 34.00$

Copyright (C) 2008 by The American Association for Thoracic Surgery doi:10.1016/j.jtcvs.2007.11.051

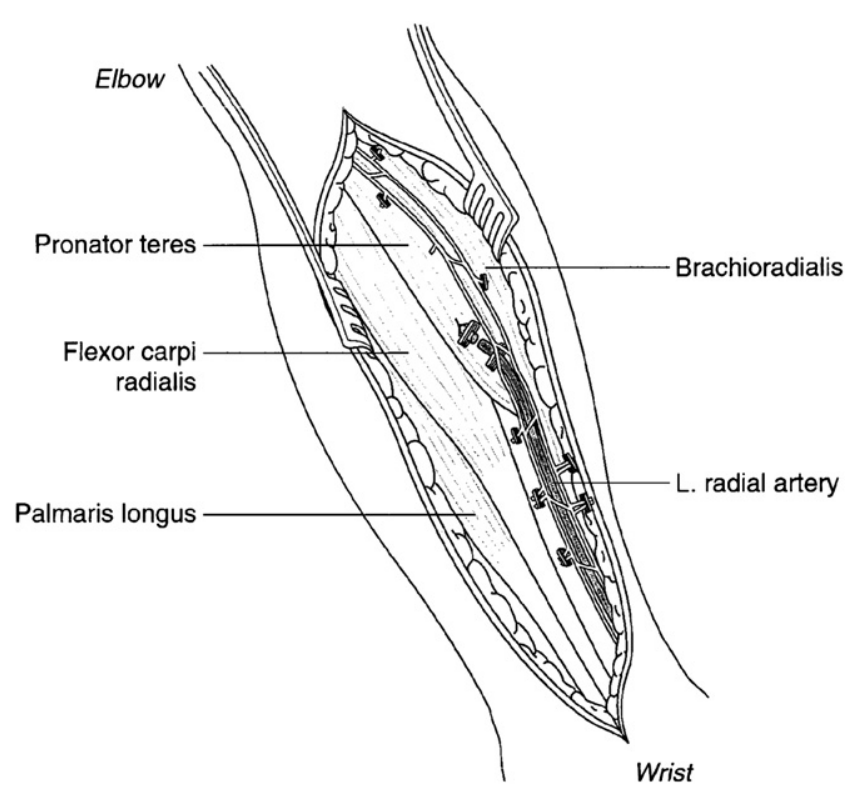

Figure 1. Schematic diagram showing the abnormal anatomic course of the left RA encountered during harvesting.

ginal arteries with the internal thoracic artery to mid-left anterior descending artery.

The patient made an uneventful recovery and was discharged on the sixth postoperative day. He was reviewed in the outpatient clinic 1 month later and had normal subjective sensation and grip power in his left forearm and hand.

\section{Discussion}

Forearm morbidity associated with RA harvesting has been reportedly low at $5 \% .^{1}$ Ischemic complications after RA harvesting are rare and usually result from insufficient ulnar collateralization of the palmar arch. ${ }^{2}$ Such congenital anomalies of forearm arteries are rare, but a knowledge of the different patterns of congenital anomalies of the ulnar and RA arteries is vital for surgeons involved in harvesting RAs. ${ }^{3}$

We have reported an abnormal anatomic course of the RA in the left forearm. Its deviation through the pronator teres muscle proximally resulted in a shortened RA that was just sufficient to bypass the diagonal 1. The formation of a "Y" graft to the left internal thoracic artery may have just reached the obtuse marginal artery.

Different variations in RA anatomy have been described. ${ }^{3}$ The most common RA abnormality is the high RA origin from the axillary artery in the upper limb, reported in approximately $15 \%$ limbs examined. ${ }^{4}$ This variation is usually associated with a normal 
anatomic course in the forearm. The anomaly we have described has not been previously reported. The closest description is by Funk and colleagues $^{4}$ of a rare anomaly arising from a distal division of the brachial artery into the ulnar and RA arteries under the pronator teres muscle with the RA pedicle assuming its normal position in the distal forearm through the pronator teres muscle. In our case, the venae comitantes had a normal anatomic course above the pronator teres. The RA in its aberrant course was not accompanied by the venae comitantes.

The development of a congenital anomaly, such as a high-origin RA, has been attributed to an arrest in stage $\mathrm{V}$ of upper limb vascular development (embryos of $23 \mathrm{~mm}$ C-R length). ${ }^{5}$ The proximal venae comitantes pedicle with the band may represent a developmental regression of the proximal part of the RA. ${ }^{5}$ As reported by Funk and colleagues, ${ }^{4}$ we believed that this anomaly would not be identifiable on routine preoperative examination of the forearm. Other forearm arterial anomalies have been described that are associated with hand vascular anomalies. ${ }^{2,5}$

\section{Conclusions}

A knowledge of the major arterial variation of the upper arm should be consider essential for anyone involved in harvesting of RA conduits to help avoid unnecessary difficulties or complications.

\section{References}

1. Desai ND, Cohen EA, Naylor CD, Fremes SE. A randomized comparison of radial-artery and saphenous-vein coronary bypass grafts. $N$ Engl J Med. 2004;351:2302-9.

2. Fox AD, Whiteley MS, Phillips-Hughes J, Roake J. Acute upper limb ischemia: a complication of coronary artery bypass grafting. Ann Thorac Surg. 1999;67:535-6.

3. McCormack LJ, Cauldwell EW, Anson BJ. Brachial and antebrachial arterial patterns. A study of 750 extremities. Surg Gynecol Obstet. 1953;96:54.

4. Funk GF, Valentino J, McCulloch TM, Graham SM, Hoffman HT. Anomalies of forearm vascular anatomy encountered during elevation of the radial forearm flap. Head Neck. 1995;17:284-92.

5. Gonzalez-compta $X$. Origin of the radial artery from the axillary artery and associated hand vascular anomalies. J Hand Surg [Br]. 1991;16A:293-6.

\title{
Open stent grafting of descending aorta to control suture line hemorrhage in type B dissection
}

\author{
Imran Saeed, MRCS, Athanasios Tsiouris, MRCS, Tahir Ali, MRCS, Jean-Pierre van Besouw, FRCA, Matt Thompson, FRCS, \\ and Marjan Jahangiri, FRCS, London, United Kingdom
}

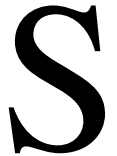
tent graft placement under direct vision has previously been described during surgical repair of type A aortic dissection to manage further entry flaps or dissection of the descending aorta. ${ }^{1,2}$ In these series, stents are deployed in an antegrade fashion with the aim of inducing thrombosis of the false lumen and pre-empting possible future complications that may arise from residual type B dissection. Kubota, Endo, and Sudo $^{3}$ have described techniques to facilitate the insertion of stents in this manner and make it safer.

We describe the use of retrograde open stent graft placement as a rescue procedure to control significant suture line hemorrhage after open repair of a descending aortic dissection.

\section{Clinical Summary}

A 29-year-old man with Marfan syndrome who had undergone aortic root replacement with a Freestyle prosthesis (Medtronic, Inc,

From the Departments of Cardiothoracic and Vascular Surgery, St George's Hospital, London, United Kingdom.

Received for publication Dec 17, 2007; revisions received Jan 23, 2008; accepted for publication Feb 2, 2008.

Address for reprints: Marjan Jahangiri, FRCS, Department of Cardiac Surgery, St George's Hospital Medical School, Blackshaw Rd, London SW17 0QT, United Kingdom (E-mail: marjan.jahangiri@stgeorges.nhs.uk).

J Thorac Cardiovasc Surg 2008;135:1390-2

$0022-5223 / \$ 34.00$

Copyright (C) 2008 by The American Association for Thoracic Surgery doi:10.1016/j.jtcvs.2008.02.039
Minneapolis, Minn) and only proximal aortic replacement 2 years previously at another hospital arrived at our emergency department with right hypochondrial pain. Computed tomographic (CT) scans showed a 9-cm infrarenal abdominal aortic aneurysm (AAA) and a 5 -cm descending thoracic aorta.

The patient underwent laparotomy, which revealed a ruptured juxtarenal AAA.

A significant pectus deformity associated with Marfan syndrome made it impossible to access the supraceliac aorta. Zenith stents (Cook Inc, Bloomington, Ind) were, therefore, deployed to cover the AAA, both iliac arteries, and the left renal artery.

After this, the patient made satisfactory progress. However, CT scans a week after the operation showed a type I endoleak and a new dissection in the descending aorta. The dissection extended from the level of the pulmonary trunk to just above the previously inserted abdominal stent graft. The diameter of the descending aorta was $5.3 \mathrm{~cm}$ and was noted to have an acute "U-bend" just above the diaphragm, rendering endovascular repair difficult. The endoleak was managed with a Palmaz stent (Johnson \& Johnson Interventional Systems, Warren, NJ) inserted via the brachial approach, and the dissection of the descending aorta was managed medically. The size of the descending aorta remained stable and the patient was discharged home.

He returned a month after discharge with back pain. CT scans at this point showed progressive expansion of the descending aorta to $6.5 \mathrm{~cm}$ associated with a moderate-sized left pleural effusion and a proximal intramural hematoma (Figure 1). Considering these new findings, we proceeded with open repair of the descending aorta. 